\title{
HYPOCHONDRIACAL ATTITUDES, PAIN SENSITIVITY, AND ATTENTIONAL BIAS
}

\author{
Paul Pauli, Michael Schwenzer, Stuart Brody, Harald Rau and \\ Niels Birbaumer
}

(Received 9 February 1993; accepted in revised form 21 April 1993)

\begin{abstract}
The relation between hypochondriacal attitudes, thermal pain threshold, and attentional bias toward pain was examined in a non-clinical population $(N=28)$. Attentional bias was operationalized with a concentration-performance test, which subjects performed while connected to a pain stimulator. Subjects were informed that they would receive a painful stimulus during the second part of the test, while the first part was introduced as pain-free. The pain stimulus was never applied during the test phase. The expectancy of a forthcoming pain stimulus reduced the performance of high hypochondriacal subjects in both parts of the test. Low hypochondriacal subjects, on the other hand, displayed significantly better performance in the first, pain-free compared to the second, pain-related part of the test. Thermal pain thresholds were assessed at four measuring sites (thenar, neck, collar-bone, abdomen), but no relations with hypochondriasis sum scores and locus of pain stimulation were found. A stepwise multiple regression of pain threshold by individual lllness Attitude Scales (IAS) led to $66 \%$ of the variance being explained by the scales 'concern about pain', 'worry about illness', and 'disease phobia'. Results are discussed in terms of amplifying somatic style, preoccupation with or attentional bias toward bodily symptoms, and experimental induction of a hypochondriacal state.
\end{abstract}

\section{INTRODUCTION}

ONE WAY of understanding hypochondriasis is as an amplifying somatic style [1] . It is assumed that hypochondriacs augment normal body sensations, and for this reason experience normal bodily sensations as more noxious and more intense than non hypochondrial subjects. Studies found that hypochondriasis or disease phobia is associated with lowered thresholds for electrical [2] or ischemic pain [3], heightened perceptual sensitivity [4], increased awareness of cardiac activity [5], somatosensory amplification [6], and enhanced sensitivity to illness cues [7, 8]. Other studies indicate that subjects characterized as high monitorers or sensitizers showed more hypochondriacal complaints, reported more physical symptoms, and displayed more abnormal illness behavior than low monitorers or repressors [9]. Reinforcement from family members, friends, or care-takers could play an important role in the development and maintenance of an amplifying somatic style and associated illness behavior. The family is believed to be particularly important in reinforcing hypochondriacal attitudes and behavior [1].

Attention is seen as a factor able to alter the perceived intensity and level of distress associated with several bodily processes including pain [10], and one could assume that hypochondriacal subjects focus more attention on bodily sensations [ 1 , 11,12 ]. However, no studies so far have examined attentional bias towards aversive bodily sensations in hypochondriacal subjects. Ahles et al. [13] tried to investigate the effect of attention on the perception of pain in subjects with high and low body

Address for correspondence: Dr Paul Pauli, Institut für Medizinische Psychologie und Verhaltensneurobiologie, Gartenstr. 29, D-7400 Tübingen, Germany. 
consciousness by asking the subjects to rate pain experiences while sitting in front of a mirror. This manipulation had no effects, which the authors blame on the inadequacy of the method. They conclude that the use of a more physiologically relevant manipulation of attention would be more appropriate.

The present study was designed to evaluate the relation between hypochondriacal attitudes, pain threshold, and attentional bias. Thermal heat was used as a pain model. This stimulus is known to activate slow conducting $\mathrm{C}$-fibers, which seem to mediate affective components of pain $[14,15]$. To evaluate attentional bias towards bodily sensations, a pain stimulus was announced while subjects performed an attention-concentration test. The effect of this manipulation on test performance was used as marker for attentional bias. Hypochondriacal attitudes and illness behaviour were assessed with the 'Illness Attitude Scales [8] Two hypotheses were tested. subjects with high hypochondriasis scores were expected (1) to exhibit low pain thresholds, and (2) to show an attentional bias towards an expected pain stimulus resulting in a reduced performance in an attention-concentration test.

Twenty-two female and 6 male right-handed subjects age $20-40(27.2 \pm 4.8)$ were studied. One female subject was excluded because of missing data. Subjects were undergraduate psychology students who received credit for participation. All were informed prior to the experiment that they would receive thermal stimuli at their pain threshold, and that they could terminate the experiment at any time without negative consequences. All subjects signed a consent document in accord with the Helsinki declaration

Apparatus and pain threshold assessment

Cutaneous heat stimuli were applied with the Path-Tester MPI-100 from Phywe Systeme GmbH Göttingen, Germany. The device controls a Marstock thermode that functions on the Peltier principle and can be both heated and cooled. The baseline temperature was $38^{\circ} \mathrm{C}$ during the pain threshold measurement and the rate of heating and cooling was set to $0.7^{\circ} \mathrm{C} / \mathrm{s}$. The stimulating area of the thermode is $1.6 \times 3.6 \mathrm{~cm}^{2}$. Galfe et al. [14] provides technical information and detai] on the accuracy and reliability of the method.

During the experiment the subjects sat in a comfortable chair in a sound-attenuated room. For pain threshold assessment, the thermode was mounted on an articulated arm and could be easily positioned at any measurement site. A spring kept the thermode pressed against the skin with a pressure of approximately $0.4 \mathrm{~N} / \mathrm{cm}^{2}$. The response panel was placed in front of the subject, who could easily press the response key with the index finger of the right hand. During each trial, the thermode was heated, and the subject had to press the response button as soon as pain was perceived. The thermode was then actively cooled to the baseline temperature. This procedure was repeated eight times at each measure ment site. The inter-trial interval was $10 \mathrm{sec}$. Each trial was announced by a beep, and the temperature started to rise after a (pseudorandomly determined) delay between. 1 and $3 \mathrm{sec}$. On average, the eight pain stimuli were delivered within $4 \mathrm{~min}$. The first three trials were run in order to adapt the subjects to the temperature of the thermode. The mean of the last five trails was considered to be the actual pain threshold at a given measurement site.

For pain measurement at the thenar and for conducting the concentration-performance tests, a smail table was mounted in front of the subjects. In these cases, the thermode came out through a square hole at the left side of the table, so that subjects could place the thenar of their left hand on the thermode. In this position it was possible to freely use the right hand while the left was in constant contact with the thermode. During the concentration test, subjects were instructed to keep their left hand on the thermode, while the response panel was removed. The experimenter supervised compliance of subjects. In this case, thermode temperature was kept constant at $38^{\circ} \mathrm{C}$

\section{Procedure}

After arriving in the laboratory, subjects received written instruction about the course of the experiment. It was emphasized that the purpose of the experiment was not to measure how much pain subjects could tolerate, but to determine when the first painful sensation occurs. After collecting subjects personal 
data, pain thresholds were determined at the thenar of the left hand (thenar), at the neck just left of the spine (neck), at the chest directly under the left collar-bone (collar-bone), and at the abdomen just under the lowest left rib (abdomen). The concentration-performance test was administered while subjects kept their left hand on the thermode of the pain stimulator. Before the test began, subjects were informed that a thermal stimulus above the pain threshold would be delivered during the second half of the test. In the middle of the test, subjects were shortly interrupted, and the experimenter performed a manipulation at the computer controlling the pain stimulator. However, this manipulation was faked, and subjects did not receive any pain stimulus during the concentration test. Finally, subjects completed some psychological tests, and were then interviewed about past and present illnesses and medical treatments.

We actually did not deliver a pain stimulus during the concentration performance test for several reasons. Firstly, the same pain stimulus has different effects (i.e. perceived aversiveness) on different subjects, and therefore observed attentional bias could be due to these interindividual differences. Secondly, an adjustment of the pain stimulus to individual pain thresholds (i.e. $1^{\circ} \mathrm{C}$ above previously determined pain threshold) would have produced pain stimuli of different duration because of constant rate of heating $\left(0.7^{\circ} \mathrm{C} / \mathrm{sec}\right)$. Thirdly, we wanted to assess attentional bias only related to psychological manipulations and independent of the physical properties of the pain stimulus. The decision not to deliver a pain stimulus made it impossible to balance the sequence of expected pain stimuli (first vs second half of the test), because the announcement of a pain stimulus in the first half without delivering it could have irritated subjects and adversely affected the latter part of the measure.

\section{Concentration-performance test}

Concentration-performance was measured with the ' $d 2$ Aufmerksamkeits-Belastungs-Test' [16], consisting of 14 lines with 47 letters each. The target was the letter ' $d$ ' with two dashes (either two dashes above or below the ' $d$ ', or one above and below the ' $d$ '), and distractors were the letter ' $d$ ' with one, three or four dashes, or the letter ' $p$ ' with any number of dashes. The subjects' task was to read through each line and to mark all targets while ignoring the distractors. Time pressure was induced by allowing the subject to work at each line for only $20 \mathrm{sec}$. Twenty-second periods were announced by the experimentor, who also checked that subjects really switched to the next line. After explaining the test. subjects completed one practice line in order to get used to the task. Errors within this line were corrected by the experimentor. Then subjects received the following instruction: 'You should perform this test while your left hand is on the pain stimulator. The test consists of 14 lines. There will be no pain stimulus until you reach line 7 . However, as soon as you stan line 8 , I will switch on the computer, and you will receive a painful stimulus sometime between line 8 and line 14 . The time will be determined by chance by the computer. We know your pain threshold from the former measurements, and the nex stimulus will be above the pain threshold. You cannot avoid the painful stimulus, but work as quickly and as exactly as possible."

Performance of subjects was determined independently for the first (no pain expected) and the second half of the test (Pain expected). The performance score corresponds to the total number of monitored letters minus the mistakes (overlooked ' $d$ ' with two dashes plus wrongly marked letters) divided through the number of completed lines.

\section{Psychological assessment.}

The Illness Attitude Scales (IAS) [8, 17] (German translation by the authors) were used to assess abnormal illness behavior and hypochondriasis. The test contains nine scales, each consisting of three questions. Questions are self-rated on five-point rating scales (no, rarely, sometimes, often, most of the time), scored 0 through 4 . The highest possible score for each scale is 12 . The scales are: (1) worry about illness, with questions such as 'Are you worried that you will get a serious disease in the future?' (2) concerns about pain. e.g. 'If you have a pain do you worry that it may be caused by a serious illness?' (3) health habits, e.g., 'Do you examine your body to find out whether there is something wrong?' (4) hypochondriacal beliefs, e.g., 'Do you believe that you have a physical disease but the doctors have not diagnosed it correctly?' (5) thanatophobia, e.g. 'Are you afraid of news that reminds you of death (such as funerals or obituary notices)?' (6) disease phobia, e.g. 'Are you afraid that you may have cancer?' (7) bodily preoccupation, e.g., 'When you feel sensations in your body, do you worry about them?' (8) treatment experience, e.g., 'How often do you see a doctor?' (9) effects of symptoms, e.g. 'Do your bodily symptoms stop you from working?". According to Kellner [8], most hypochondriacal patients can be identified by means of two scales, the disease phobia and the hypochondriacal beliefs scales. A score of either three or four on one of the related subscales indicates hypochondriasis. The frequency of these responses was assessed in our sample of psychology students. In order to get a continuous measure of hypochondriacal attitudes, a hypochondria sum score of these two scales was also calculated. By means of a median split, two groups of subjects (with high and low hypochondriacal attitudes) were formed. 
The Freiburger Beschwerdenliste (FBL) [18] was administered to evaluate the subjects bodily complaints. This scale consists of 78 items, covering various sources of bodily complaints (gastrointestinal, muscular, cardiovascular, skeletal, etc.). Frequency of complaints are self-rated on a fivepoint scale with the following cues: never, about twice a year, about twice a month, about three times a week, or daily, scored 0 through 4. A sum score reflects the total amount of experienced bodily complaints. Three scales out of the Freiburger Persönlichkeitsinventar (FPI-R) [19] were used to assess neuroticism, extraversion, and stress.

\section{Interview}

In a semi-structured interview, subjects were asked about their current and former illnesses, and about serious illnesses in the family. A rating scale $(0$ to 6$)$ was used to assess the way their parents cared for them when they were ill.

Statistical analysis

Parametric tests (t-tests, ANOVA) were performed to analyze differences between low and high hypochondriacial subjects Significant ANOVA effects were further analyscd with post-hoc means comparisons. A stepwise multiple regression analysis was performed to examine which psychological scales contribute to the variance of the pain threshold. Statistical significance was set at $5 \%$ for all tests.

\section{Hypochondriasis}

\section{RESULTS}

In our sample of psychology students, two subjects were identified with scores of three or four on one of the hypochondriacal beliefs or the disease phobia subscales, which is assumed to be a characteristic response for subjects with hypochondriasis [8]. Kellner et al. [20] used the same test criteria, and found five subjects fulfilling these criteria in two samples of 60 medical and law students.

A median split according to the hypochondria sum score (disease phobia plus hypochondriacial attitudes median $=2$ ) led to groups of subjects with high and low hypochondriacal attitudes. Table $I$ depicts that these two groups are roughly

TABIE 1.-CHARACTERISTICS OF HIGH AND LOW HYPOCHONDRIACAL SUBJECTS (MEANS \pm SD)

\begin{tabular}{lccc} 
& \multicolumn{2}{c}{ Hypochondriacal attitude } \\
& Low & High & Test \\
\hline Female/male $(N)$ & $12 / 4$ & $9 / 2$ & Chi ${ }^{2}=0.2 \mathrm{NS}$ \\
Age & $27.6 \pm 5.6$ & $26.2 \pm 3.3$ & $t=0.8 \mathrm{NS}$ \\
Weight (kg) & $59.9 \pm 13.2$ & $58.3 \pm 9.5$ & $t=0.1 \mathrm{NS}$ \\
Height (cm) & $169.6 \pm 8.0$ & $169.2 \pm 9.4$ & $t=0.7 \mathrm{NS}$ \\
Childhood illness (N) & $1.7 \pm 1.2$ & $2.3 \pm 3.0$ & $t=0.9 \mathrm{NS}$ \\
Parents care during illness & $4.9 \pm 1.1$ & $5.0 \pm 1.2$ & $t=0.1 \mathrm{NS}$ \\
$\quad(0-6)$ & $3.8 \pm 2.2$ & $5.6 \pm 3.7$ & $t=1.7 \mathrm{NS}$ \\
Stress (FPI-R) & $5.9 \pm 2.7$ & $7.1 \pm 2.8$ & $t=1.1 \mathrm{NS}$ \\
Extraversion (FPI-R) & $6.4 \pm 3.0$ & $8.1 \pm 2.8$ & $t=1.5 \mathrm{NS}$ \\
Neuroticism (PFI-R) & $88.1 \pm 26.2$ & $108.7 \pm 30.4 t=1.9 p=0.07$ \\
Bodily complaints (FBL) & & & \\
Illness attitude scales (1AS) & $3.3 \pm 1.5$ & $6.5 \pm 2.2$ & $t=4.4 p=0.0002$ \\
Worry about illness & $3.8 \pm 2.1$ & $5.8 \pm 2.0$ & $t=2.4 p=0.02$ \\
Concern about pain & $6.2 \pm 2.7$ & $6.3 \pm 2.5$ & $t=0.1 \mathrm{NS}$ \\
Health-habits & $0.1 \pm 0.3$ & $2.5 \pm 2.2$ & $t=4.4 p=0.0002$ \\
Hypochondriacal beliefs & $2.6 \pm 2.4$ & $4.4 \pm 2.1$ & $t=1.9 p=0.07$ \\
Thanatophobia & $1.1 \pm 0.6$ & $3.7 \pm 2.5$ & $t=4.0 p=0.0005$ \\
Disease Phobia & $3.1 \pm 1.4$ & $5.0 \pm 2.5$ & $t=2.6 p=0.02$ \\
Bodily preoccupation & $4.3 \pm 1.8$ & $4.6 \pm 2.7$ & $t=0.4 \mathrm{NS}$ \\
Treatment experience & $2.5 \pm 1.9$ & $4.7 \pm 2.5$ & $t=2.6 p=0.01$ \\
Effects of symptoms & & & \\
\hline
\end{tabular}


comparable in sex ratio, age, weight, height, number of remembered childhood illnesses, intensity of parents care during illness, stress, neuroticism, and extraversion. The bodily complaints of the high hypochondriasis group are somewhat greater $(p=0.07)$, which validates the group formation.

\section{Attentional influences}

The expectation of the pain stimulus was associated with an attentional shift $(F(1,25)=7.4, p=0.01)$, which affected high and low hypochondriacal subjects in different ways (interaction: $F(1,25)=5.0, p=0.03$ ). Figure 1 depicts that both groups showed relatively poor performance when a pain stimulus was expected. However, subjects with low hypochondriasis sum scores performed significantly better in the 'pain-free' interval $(t(15)=4.1, p=0.001)$, whereas high hypochondriacal subjects displayed a consistently poor performance $(t(10)=0.3$, NS).

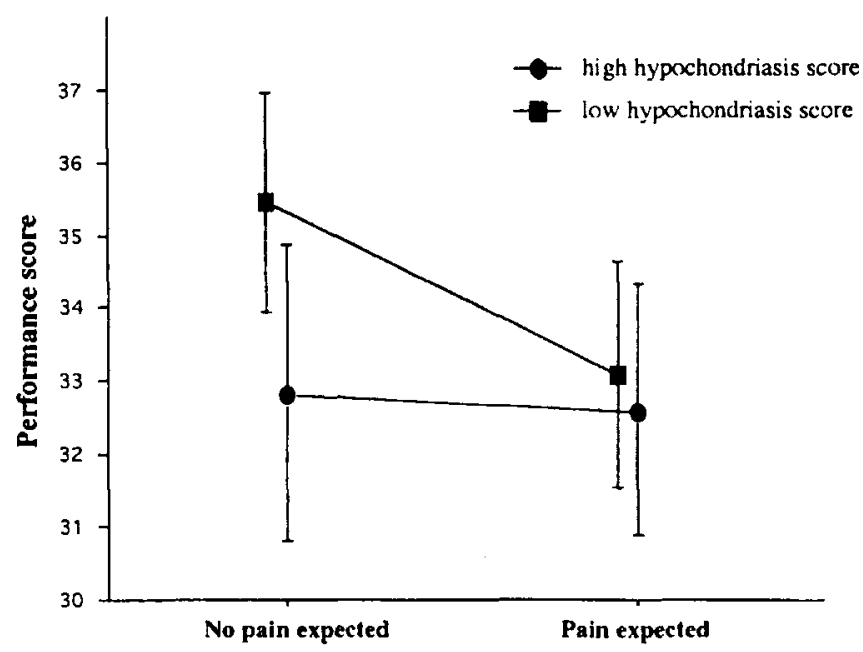

Fig. 1. Performance in a concentration-performance test while high and low hypochondriacal subjects are connected to a pain stimulator (for details see text; means and standard errors are presented).

\section{Pain threshold}

The pain thresholds differed according to location $(F(3,75)=8.1, G-G=0.87$, $p=0.0002$ ), but were not affected by the subject's hypochondriasis sum score $(F(3,75)=0.2$, NS) (see Fig. 2). Significant threshold differences were found between the thenar and the abdomen $(t(26)=2.9, p=0.008)$ or the neck $(t(26)=$ $2.7, p=0.01$, but not between the thenar and the collar-bone $(t(26)=1.3$, NS).

\section{Correlations and regression analysis}

A significant negative correlation between thenar pain threshold and the IAS scale 'concern about pain' $(r=-0.39, p=0.04)$ was found. Negative correlations between thenar pain threshold and the IAS scales 'worry about illness' $(r=-0.33, p=0.09)$ and 'treatment experience' $(r=-0.36, p=0.06)$, and for the rating 'parents care during illness' $(r=-0.37, p=0.06)$ approached significance. 


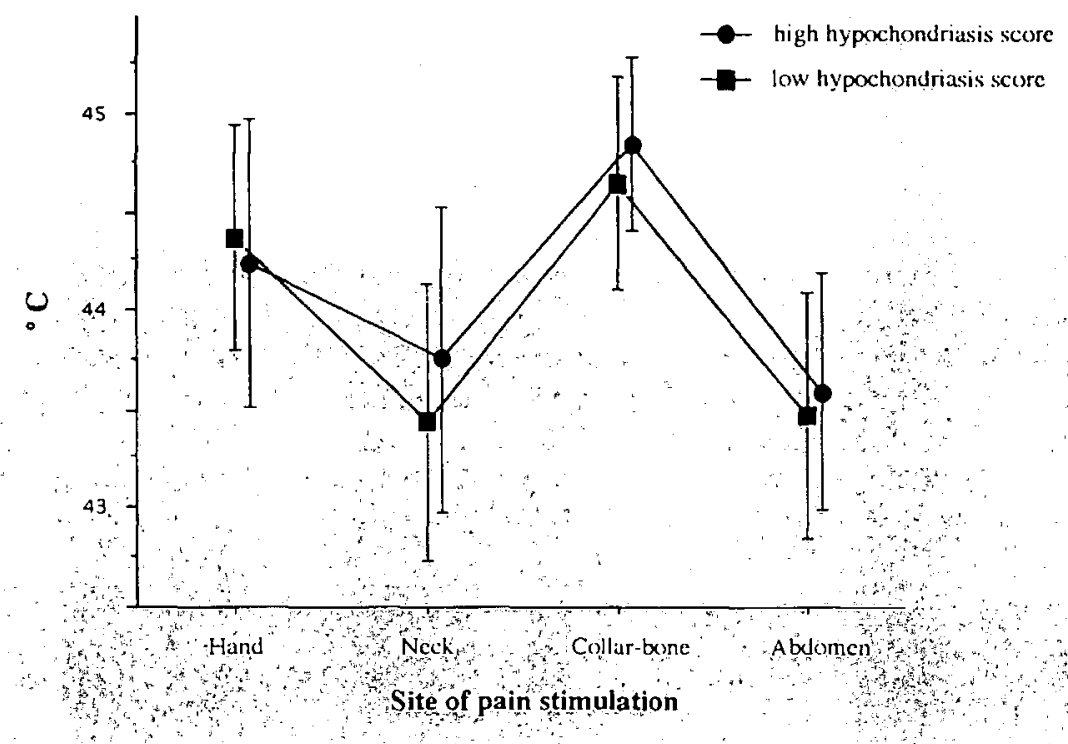

Fig. 2. Thermal pain threshold of high and low hypochondriacal subjects at four measurement sites (means and standard errors are presented).

A stepwise multiple regression analysis with pain threshold at the thenar as dependent variable and the IAS scales the FPI-R scales, the FBL sum score, the rating about the parents care during illness, and the number of childhood ilnesses as predictor variables was performed to determine psychological predictors for pain threshold. Results are summarized in Table II: Three IAS-variables, concern about pain', 'disease-phobia', and 'worry about illness' entered the equation, accounting for $66 \%$ of the variance of the pain threshold at the thenar.

TABLE II.-STEPWISE MULTIPLE REGRESSION OF PAIN THRESHOLO BY Illoness ATtítude SCALES

\begin{tabular}{llrrr}
\hline Step & Variable & \multicolumn{1}{c}{$\mathrm{b}^{*}$} & \multicolumn{1}{c}{$R^{2}$} & $\rho$ \\
\hline 1 & Concern about pain & -0.27 & 0.15 & 0.04 \\
2 & Disease phobia & 1.08 & 0.31 & 0.01 \\
3 & Worry about illness & -0.89 & 0.66 & $<0.0001$ \\
\hline
\end{tabular}

*b are those obtained at the last step

\section{DISCUSSION}

The illness attitudes scales (IAS) scores of the subjects are in general comparable with the scores of the student populations examined by Kellner et al. [20]. Two out of the 30 students fulfilled the criteria of Kellner [8] for hypochondriasis, which is consistent with Kellner's observed incidence.

High and low hypochondriacal subjects did not differ in pain thresholds. A comparison with the normative data of Lautenbacher et al. [21] for the pain threshold at the thenar reveals that both groups' pain thresholds are in the normal range. 
However, the announcement of a pain stimulus at the end of an attention-concentration test had very different effects on the performance of high or low hypochondriacal subjects. While both groups revealed an attentional bias towards the pain stimulus in the 'pain-expected' epoch, only low hypochondriacal subjects displayed a distinctly better performance in the preceding 'pain-free' epoch. The performance of high hypochondriacal subjects was poor during the whole test, suggesting a possible attentional bias or preoccupation toward painful sensations.

At first glance, one might speculate the high hypochondriacal subjects' performance in the attention-concentration test is in general so poor that even the announcement of a pain stimulus cannot depress their performance further. However, this 'floor-effect' explanation is unlikely. The variance of the performance score of both groups is very similar, and a true floor effect would have limited variance (see Fig. 1). A comparison with the norm data of Brickenkamp [16] reveals that the performance of our subjects was quite comparable with college educated subjects in those normative data, and even the high hypochondriacal subjects' mean score was above the 40th percentile. Compared with the general population between age 19 and 40 , our subjects mean performance was above the 90th percentile (the worst performing subject was at the 42 nd percentile). There is no reason to assume that a further deterioration in high hypochondriacal subjects test performance would not have been detected.

The results indicate that in high hypochondriacal subjects an attentional shift toward a possible pain stimulus is a trait characteristic. This interpretation fits with the assumption of Barsky and Klerman [1] that the augmention of bodily symptoms in hypochondriasis is mediated by a specific and constant attentional bias, and the formulation of Warwick et al. $[12,22]$ that an increased focus on bodily sensation is a characteristic of hypochondriasis. There are two explanations for the observed attentional bias in hypochondriasis, both of which may be operative. First, high hypochondriacal subjects ignore or do not believe safety signals and are not able to relax when given information about pain free intervals. Secondly, the announcement of an unavoidable pain stimulus leads to an experimentally induced hypochondriasis in low hypochondriacal subjects.

Correlation analysis revealed that pain thresholds are not independent from hypochondriacal attitudes and illness behavior. The highest direct correlation $(r=-0.39)$ was found with the IAS scale 'concern about pain', meaning that subjects with high concerns have lower pain thresholds. Additionally, marginal significant correlations with 'worry about illness', 'treatment experience' and 'parents care during illness' all pointed in the expected direction, indicating that a low pain threshold is associated with increased worry about illness, increased illness behavior, and reinforcement of illness from parents. A stepwise regression analysis showed that three IAS scales ('concern about pain', 'disease phobia', 'worry about illness') were sufficient to explain $66 \%$ of the pain threshold's variance. A strong relation between psychological variables and the psychophysiological variable pain threshold has to be assumed. Barsky and Klerman [1] propose that hypochondriasis corresponds to an 'amplifying somatic style'. In general, our data support this assumption. It seems that a specific combination of several hypochondriacal attitudes is to a large degree responsible for an increased sensitivity for painful stimuli. This can also explain why most studies found only modest correlations (from $r=0.15$ to $r=0.30$ ) between 
pain thresholds and global, unidimensional measures of hypochondriasis [2, 3] .

A seeming limitation of the present study is the use of a non-clinical population.

However, as noted by Warwick [12], the same issues determining hypochondriasis are pertinent to non-clinical populations'-somatic focus. Our use of exclusively nonclinical subjects may actually augment rather than diminish the significance of the findings, as the variance of hypochondriasis was limited, reducing the likelihood of detecting differences.

Acknowledgements - Research was supported by the Deutsche Forschungsgemeinschaft (Forschergruppe Klinische Psychophysiologie des Schmerzes and by NBN Medizin Elektronik München.

\section{REFERENCES}

1. BARSKY AJ, KLERMAN, GL Overview: Hypochondriasis bodily complaints and somatic styles Am $J$ Psychiat 1983; 140: 273-283

2. BIANCh! GN. Origins of disease phobia. Aust $N Z$ J Psychiat 1971; 5: $241-257$.

3. Ziesat HA. Correlates of the toumiquet ischemia pain ratio. Percept Moror Skills $1978 ; 47: 147-150$

4. WAGNER HJ. REYELLE, W. Arousal and perceptual sensitivity in hypochondriacs $J$ Abnorm Psychol 1978; 87:523-530.

5. TYRER P, LEE I, ALEXANDER J. Awareness of cardiac function in anxious, phobic and hypochondriacal patients Psychol Med 1980; 10: $171-174$

6. BARSKY AJ. WYSHAK G. Hypochondriasis and somatosensory amplification. Br J Psychiat 1990 157: $404-409$

7. HrснсоOK PB MATHEWs, A Interpretation of bodily symptoms in hypochondriasis Beht Res Ther 1992:30: $223-234$

8. Kellner R. Somatization and Hypochondriasis New York Praeger Greenwood 1986

9. MLLER MS, BRODY DS, SUMMERTON J. Styles of coping with threat implications for health. J Person Soc Psychol 1988;54:142-148:

10. Miltner W. Johnson R, Braun C, LARBig W Somatosensory event-related potentials to painful and non-painful stimuli: effects of attention. Pain $1989 ; 38: 303-312$

1 1. PENNEBAKeR JW. The Psychology of Physical Symptoms. New York: Springer, 1982

12. WARWICK HMC. A cognitive-behavioural approach to hypochondriasis and health anxiely J Psychosom Res 1989; 33: 705-711.

13. Ahles TA, Cassens HL, Stalling RB. Private body consciousness, anxiety and the perception of pain. J Behav Ther Exper Psychiat 1987; 18: 215-222.

14. Galfe G, Lautenbacher S, Hölzl R. Strian F. Diagnosis of small-fibre neuropathy; computerassisted methods of combined pain and thermal sensitivity determination. Hospimedica $1990 ; 8(7)$ $38 \div 48$.

15. Bromm B. Evoked cerebral potentials and pain. In Advances in Pain Research and Therapy (Edited by Fields HL, DubNeR R, CERvero F.), (vol 9) pp. 305-329. New York: Raven Press 1985.

16. Brickenkamp R. Test d2 Aufmerksamkeits-Belastungs-Test. Göttingen: Hogrefe, 1978.

17. Kellner R, AbbotT P, Winslow WW, Pathak D. Fears, beliefs, and attitudes in DSM-III hypochondriasis. J Nerv Ment Dis 1987; 175: 20-25:

18. FAHRENBERG J. Die Freiburger Beschwerdenliste FBL. Zeitschrifi für klinische. Psychologie 1975; 4: $79-100$.

19. Fahrenderg J, Hampel R, Selg H. Das Freiburger Persönlichketsinventar FPI, revidierte Fassung FPl-R und teilweise geänderte Fassung FPI-Al Göttingen: Hogrefe, 1984.

20. Kellner R, Wiggins RG, PathaK D. Hypochondriacal fears and beliefs in medical and law students. Arch Gen Psychiat 1986; 43: 487-489.

21. Lautenbacher S, Galfe G, Möltner A, Strian F. Alterskorrigierte Normdaten für die bestimmung der Temperatur- und Schmerzempfindlichkeit mit dem MPI-100 Path-Tester. MaxPlanck-Institut für Psychiatrie, 1989.

22. Warwick HC, Salkovskis PM. Hypochondriasis. Behav Res Ther 1990; 28: 705-711. 